\title{
Local tomography daubechies bases Tomografía local con bases daubechies
}

\author{
Ricardo De Armas C. ${ }^{1}$ \\ Amed Alfonso $\mathrm{C}^{2}$ \\ Lucio Rojas $C^{3}$
}

Fecha de envío: 28 de septiembre de 2015
Fecha de recepción: 15 de octubre de 2015
Fecha de aceptación: 2 de noviembre de 2015

\begin{abstract}
The Mesoamerican Information Highway (MIH) is a dialogue and coordination mechanism that articulates cooperation, development and integration efforts among the Central American countries with the purpose of improving the quality of life of the inhabitants of the region. This research paper was made with the purpose of giving the reader a state of art about the Mesoamerican Project (MP) in order to review the past, present and future situation of these kinds of technological developments in the region and their impact on the Colombian case. It shows a map in order to lead the implementation of future projects of the Information and Communications Technology (ICT) and its implementation through those alternatives.
\end{abstract}

Keywords: Local Tomography, Daubechies bases, filtered back projection, vanishment moments, Hilbert transform, Calderón-Zygmund operator.

Resumen: Este artículo explica que las bases Daubechies puede ser usadas para diseñar algoritmos de reconstrucción localizada de imágenes tomográficas desde las proyecciones almacenadas en matrices de datos dispersos. Los desarrollos de tales algoritmos reducen significativamente la cantidad de exposición a los rayos $\mathrm{X}$ trasmitidos en la tomografía, evitando daños colaterales a largo

* BSc. In Mathematics, Universidad Distrital Francisco José de Caldas, Colombia. MSc. In applied Mathematics, Universidad EAFIT, Colombia. Current position: professor at Universidad Central and Universidad de La Salle, Colombia. E-mail: rde armasc@ucentral.edu.co.

** BSc. In Mathematics, Universidad Distrital Francisco José de Caldas, Colombia. MSc. In applied Mathematics, Universidad EAFIT, Colombia. Current position: professor at Universidad Central and Universidad Militar "Nueva Granada", Colombia. E-mail: amed0808708@ucentral.edu.co.

*** BSc. In Mathematics, Universidad Distrital Francisco José de Caldas, Colombia. MSc. In applied Mathematics, Universidad EAFIT, Colombia. Current position: professor at Universidad Central and Universidad Militar "Nueva Granada”, Colombia. E-mail: lucio.rojas@unimilitar.edu.co. 
plazo en pacientes, en órganos como pulmones, corazón, y también en lesiones de la médula.

Palabras clave: Tomografía local, bases Daubechies, retroproyección filtrada, momentos de desvanecimiento, transformada de Hilbert, Operador CalderónZygmund.

\section{Introduction}

Is it possible to reconstruct a tomographic image using only integrals passing through a certain region of interest of the patient? Answering this question is to solve the problem of local tomography. A classic method implemented today to reconstruct a tomographic image is the filtered back-projection algorithm (filtered back-projection) [1], [2], [3]. However, this presents a number of problems when trying to reconstruct localized data because it requires all line integrals projection matrix images. The wavelet bases are special functions by which its nature and properties neutralize the effects of the filtered back-projection algorithm. Localization, smoothness, compact support, and vanishing moments of these bases are preserved during implementation [4]. The Daubechies wavelets bases are bases that have as much time to fade compact support [5]. The proposed solutions, in two dimensions, for the problem of local tomography have allowed advances in disciplines such as medicine, where in certain situations studies are performed on a region of interest, so there is no need to expose the patient with large amounts of radiation, preventing longterm side traversed by X-rays to the heart, lungs and in spinal injuries or organ damage. Furthermore, it is possible to reconstruct images with scarce data obtained from non-destructive testing of the object, as is the case with anthropological studies, in investigations of forensic sciences, archeology, and paleontology. This paper is organized as follows: in section 2 , the reconstruction of a tomograph- ic image recalling the concepts of projections, Radon transform, sinogram and filtered back-projection algorithm is reviewed. In section 3 , the interior problem or region of interest emphasizing the benefits of the solutions analyzed in different disciplines. In section 4, Hilbert operators and Calderón-Zygmund (OCZ), acting on the projections are analyzed. In section 5 , the Daubechies bases are characterized. Concrete examples of the behavior of the Hilbert transform and the Calderón-Zygmund operator some Daubechies are presented in Section 6. Finally, conclusions are presented.

\section{Tomographic Image Reconstruction}

The tomographic image reconstruction is a process that involves the concepts of projected Radon transform, sonogram, and inversion algorithms, such as filtered back-projection. Recall that a digital image is a two-dimensional light intensity function $\mathrm{f}(\mathrm{x}, \mathrm{y})$, where $x$ and $\mathrm{y}$ represent the spatial coordinates, and $f$ represents the gray level at that point. In our case, $f$ represents attenuation coefficients of the intensity of X-rays within a selected section of the patient.

\subsection{The projections}

A projection at a given angle is defined as a set of line integrals in said direction. Projection is denoted by $p_{\theta_{1}}(s)$, where $\theta$ is the angle indicating the direction of the integral, and $\mathrm{s}$ is the variable that marks the position of the 
VISION

ELECTRONICA detector for photon counting X-rays. Figure 1 illustrates two projections or as they are also known, attenuation profiles $p_{\theta_{1}}(s)$ y $p_{\theta_{2}}(s)$ [6], [7].

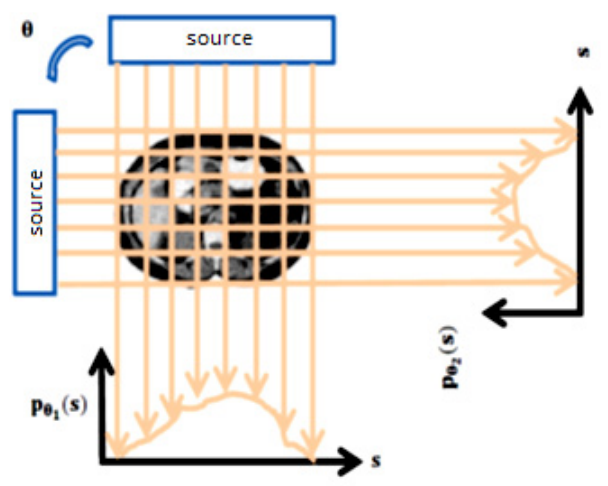

Figure 1. Projections or attenuation profiles $p_{\theta_{1}}(s)$ and $p_{\theta_{2}}(s)$. Slice an abdomen. Source own

With a sample of $\mathrm{n}$ parallel projections measures image $\left\{p_{\theta_{1}}(s) p_{\theta_{2}}(s) p_{\theta_{3}}(s), \ldots p_{\theta_{n}}(s) \quad\right.$ is possible to reconstruct the image.

\subsection{Radon's Transform}

In two dimensions, it is a transformation that maps a function $f: R^{2} \rightarrow R$ on the set of projections $\mathrm{p}_{\theta}(\mathrm{s})$ entre parentesis $x \cos \theta+y \operatorname{sen} \theta=s$. The parallel lines generated by X-rays of the tissue, from the source to the detector through the selected section of the patient, can be configured with the formula $x \cos \theta+y \operatorname{sen} \theta=s$. Here, $\mathrm{s}$ is the minimum distance from the line to the origin and $\theta$ is the angle between the $\mathrm{X}$-axis with the position vector of the point closest to the origin line, as indicated in Figure 2.

In Figure 2, two coordinate systems are also identified: the coordinate system of the patient at rest $(x, y)$ and the sample coordinate system image $\mathfrak{R}_{\theta} f(s)$, the scanner. The Radon transform denoted $\mathfrak{R}_{\theta} f(s)$ can be written in terms of the distribution $-\delta$ (delta Dirac distribution).
$\mathfrak{R}_{\theta} f(s)=p_{\theta}(s)=\int_{-\infty-\infty}^{\infty} \int_{\infty}^{\infty} f(x, y) \delta(x \cos \theta+y \operatorname{sen} \theta-s) d x d y$

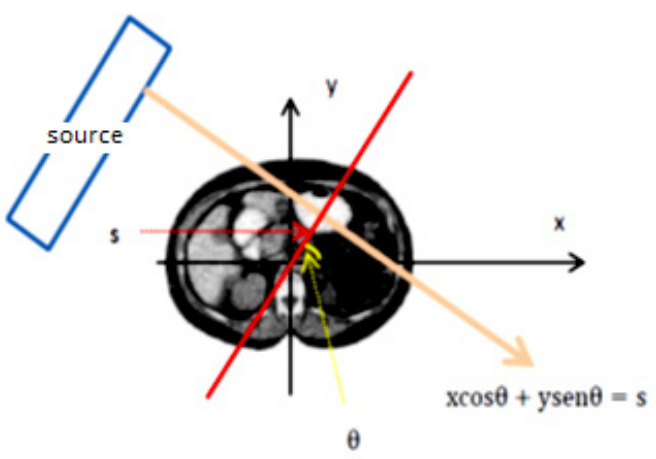

Figure 2. Lines $x \cos \theta+y \operatorname{sen} \theta=s$ through a section of the abdomen. Source: own

To reconstruct the image $f(x, y)$ from the projections, it is necessary to calculate the inverse Radon transform. There are various algorithms in the inverse Radon transform are computationally efficient for the two dimensional case. The problem in practice is that it is not possible to define the complete set of all integrals. Mathematically, the image is only determined by an infinite set of projections, not by any finite set of projections [8], [9].

\subsection{The Sinogram}

To manipulate and process the tomographic data, these are arranged in matrix form. As illustrated in Figure $3 \mathrm{~b}$ ) where the rows indicate the actual values of line integrals and the columns indicate the angles of each projection: a sonogram (the graphical representation of the matrix) is then obtained. The discrete values of the projections represent the Radon's object space. The data is only acquired from $0^{\circ}$ to $180^{\circ}$, because according to the physical symmetry, the X-ray paths behind the patient being examined do not provide different information to trajectories and steps [7]. Figure 3 illustrates the arrangement of values of the projections in the Radon space. 


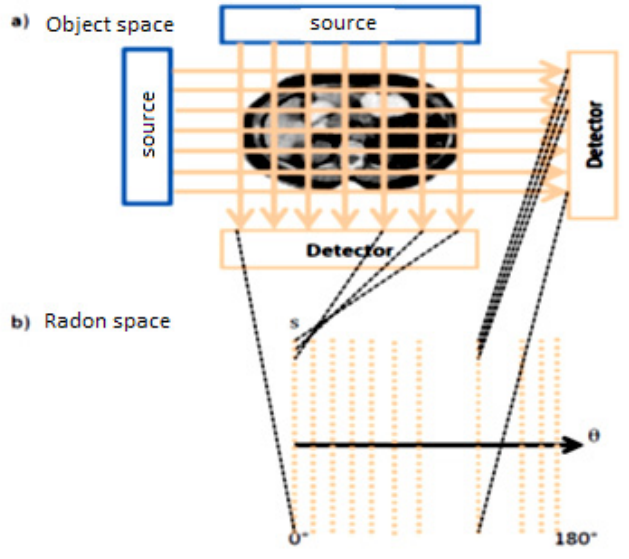

Figure 3. a) Measured values of the projections of the object in space $(x, y)$ b) Arrangement of the values of the projections in the Radon space $(\theta, s)$. Slice of abdomen. Source: own

\subsection{Filtered back-projection algorithm}

An inverse algorithm Radon transform, computationally efficient for the two-dimensional case is filtered back-projection (filtered back-projection) [1], [3], [10], [11]. This algorithm appears naturally when the coordinates are changed. For the image $F(u, v)$ from the projections, the inverse Fourier transform is applied $F(u, v)$.

$$
f(x, y)=\int_{-\infty}^{\infty} \int_{-\infty}^{\infty} F(u, v) e^{2 \pi i(x u+y v)} d u d v
$$

We change to polar coordinates,

$$
\begin{aligned}
& u=\omega \cos \theta \\
& v=\omega \operatorname{sen} \theta \\
& f(x, y)=\int_{0}^{2 \pi \infty} \int_{0}^{\infty} F(u, v) e^{2 \pi i(x \omega u+y \omega v)} d u d v
\end{aligned}
$$

Using the symmetry properties of the Fourier transform, with the absolute value notation to redefine the limits of integration:

$$
f(x, y)=\int_{0}^{\pi} \int_{-\infty}^{\infty} F(\omega \cos \theta, \omega \operatorname{sen} \theta) e^{2 \pi i \omega(x \cos \theta+y \operatorname{sen} \theta)}|\omega| d \omega d \theta(5)
$$

The court Fourier theorem allows the following change:

$$
f(\omega \cos \theta, \omega \operatorname{sen} \theta)=\hat{P}_{\theta}(\omega)
$$

Here, $\hat{p}_{\theta}(s)$ is the Fourier transform in one dimension of $p_{\theta}(s)$.

$$
f(x, y)=\int_{0}^{\pi} \int_{-\infty}^{\infty} \hat{P}_{\theta}(\omega) e^{2 \pi i \omega(x \cos \theta+y \operatorname{sen} \theta)}|\omega| d \omega d \theta
$$

Finally, denoted with $p_{\theta}^{F}(s)$ is the inner integral in (7). Then the image is reconstructed with the filtered back-projection algorithm:

$$
f(x, y)=\int_{0}^{\pi} p_{\theta}^{F}(s) d \theta
$$

This algorithm is implemented in the present, both first-generation CT scanners, as a hybrid generation in some [1], [2].

\section{Singular integral operators}

When you invert the Radon transform through the filtered back-projection algorithm, the projections can be filtered in the frequency domain or in the domain of Radon space. It is during this stage that appear acting on projections, the Hilbert transform operator and Calderón - Zygmund.

\subsection{Hilbert Transform}

The Hilbert transform of the projections $p_{\theta}(s)$ is given as:

$$
H p_{\theta}(s)=\frac{1}{\pi} \int_{-\infty}^{\infty} \frac{1}{s-\tau} p_{\theta}(\tau) d \tau
$$

To avoid the problem of singularity, the integral is calculated symmetrically [35], [36] according to the equation:

$$
\frac{1}{\pi} \int_{-\infty}^{\infty} \frac{1}{s-\tau} p_{\theta}(\tau) d \tau=\lim _{\varepsilon \rightarrow 0}\left\{\int_{-\infty}^{t+\varepsilon} \frac{1}{s-\tau} p_{\theta}(\tau) d \tau+\int_{t+\varepsilon}^{\infty} \frac{1}{s-\tau} p_{\theta}(\tau) d \tau\right\}
$$

Figure 4 illustrates the Hilbert transform of the projections, $p_{0^{\circ}}(s)$ and $p_{45^{\circ}}(s)$ of $p_{90^{\circ}}(s)$ 
Radon space ghost Shepp-Logan head. i.e. $H p_{0^{\circ}}(s), H p_{45^{\circ}}(s), \mathrm{y} H p_{90^{\circ}}(s)$.
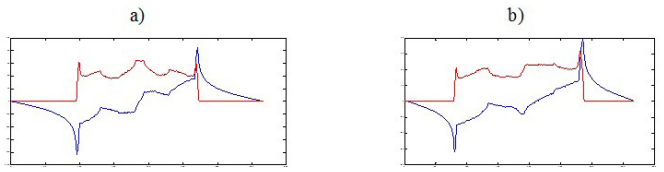

c)

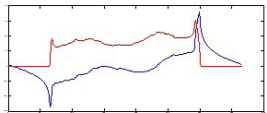

Figure 4. a) Proyection $p_{0^{\circ}}(s)$ (Red) y $H p_{0^{\circ}}(s)$ (Blue) b) Proyection $p_{45^{\circ}}(s)$ (Red) y $H p_{45^{\circ}}(s)$ (Blue) c) Proyection $p_{90^{\circ}}(s)$ (Red) y $H p_{90^{\circ}}(s)$ (Blue). Source: own

The expression (9) describes a convolution of the projection $p_{\theta}(s)$ with the function $\frac{1}{\pi s}$. i. e.:

$H p_{\theta}(s)=p_{\theta}(s) * \frac{1}{\pi s}$

Figure 5 illustrates the convolution kernel $\frac{1}{\pi s}$. This convolution kernel has a singularity at the origin and has a cross that slowly decays [4].

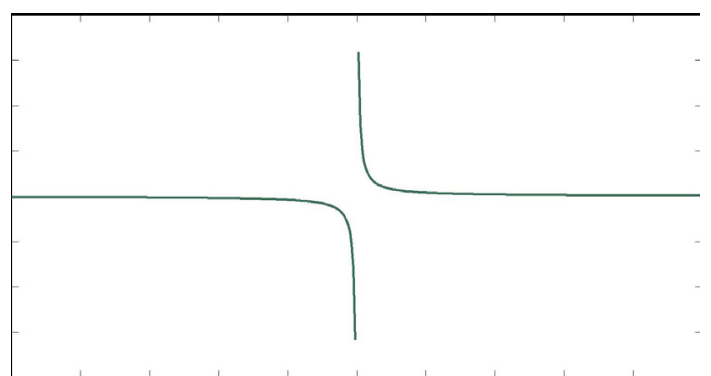

Figure 5. Convolution Kernel $\frac{1}{\pi s}$. Source: own The Fourier transform of the Hilbert transform of the projections $p_{\theta}(s)$ is,

$$
F\left\{H p_{\theta}(s)\right\}=-i \operatorname{sign}(\omega) P_{\theta}(\omega)
$$

where $P_{\theta}(\omega)$ is the one dimentional Fourier transform of $p_{\theta}(s)$ and $\operatorname{sign}(\omega)$ is the sign function defined as: $\operatorname{sign}(\omega)= \begin{cases}1, & \text { si } \omega>0 \\ 0, & \text { si } \omega=0 \\ -1, & \text { si } \omega<0\end{cases}$

\subsection{The Calderon-Zygmund operator}

The Calderón-Zygmund operator of a projection $p_{\theta}(s)$ it can be defined in terms of the Fourier transform [13], [14], as follows:

$$
\hat{T} P_{\theta}(\omega)=|\omega| P_{\theta}(\omega)
$$

Is rewritten as follows:

$$
\hat{T} P_{\theta}(\omega)=-i \operatorname{sign}(\omega)(i \omega) P_{\theta}(\omega)
$$

The multiplication by $(i \omega)$ in the Fourier domain corresponds to differentiation in the Radon domain and multiplication by $-i \operatorname{sign}(\omega)$ corresponds to the Hilbert transform [14]. In the domain of Radon operator Calderón - Zygmund denoted $T$ applied to the projections $p_{\theta}(s)$ corresponds to:

$$
T p_{\theta}(s)=H \frac{\partial p_{\theta}(s)}{\partial s}
$$

The expression (16) describes the convolution.

$T p_{\theta}(s)=\frac{1}{\pi s} * \frac{\partial p_{\theta}(s)}{\partial s}$

Figure 6 illustrates the operator Calderon Zygmund applied to the projections $p_{0^{\circ}}(s)$ , $p_{45^{\circ}}(s)$ and $p_{90^{\circ}}(s)$ Radon space ghost Shepp-Logan head. i.e., $T p_{0}{ }^{\circ}(s), T p_{45^{\circ}}(s) \mathrm{y}$ $p_{90^{\circ}}(s)$.

The convolution integral projections $p_{\theta}(s)$ with compact support, should be taken as a Cauchy principal value at a point within the support.

$T p_{\theta}(s)=\lim _{\varepsilon \rightarrow 0}\left\{\int_{-\infty}^{t-\varepsilon} \frac{1}{s-\tau} p_{\theta}(\tau) d \tau+\int_{t+\varepsilon}^{\infty} \frac{1}{s-\tau} p_{\theta}(\tau) d \tau\right\}$ 


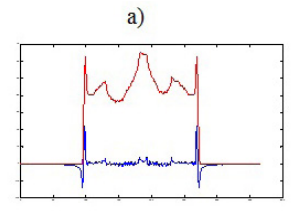

b)

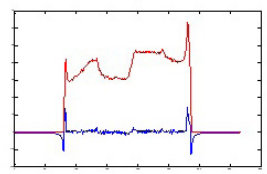

c)

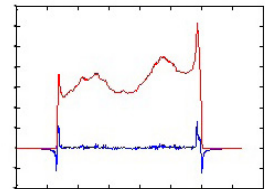

Figure 6. a) Projection $p_{0^{\circ}}(s)(\operatorname{Red})$ y $T p_{0^{\circ}}(s)$ (Blue) b) Projection $p_{45^{\circ}}(s)$ (Red) y $T p_{45^{\circ}}(s)$ (Blue) c) Projection $p_{90^{\circ}}(s)$ (Red) y $T p_{90^{\circ}}(s)$ (Blue). Source: own

For values outside the support of $p_{\theta}(s)$, the convolution integrals are ordinary integrals:

$T p_{\theta}(s)=\frac{1}{\pi} \int_{-\infty}^{\infty} \frac{1}{s-\tau} \frac{\partial p_{\theta}(\tau)}{\partial \tau} d \tau$

If $p_{0^{\circ}}(s)$ has compact support, $T p_{0^{\circ}}(s)$ will not have compact support. The Calderón Zygmund operator $\mathrm{T}$ is not local, insomuch as $\operatorname{Tp}_{0^{\circ}}(s)$, at some point, requires all values of the function $p_{\theta}(s)$.

\section{Local tomography}

In computed tomography you can find complete and incomplete sampling [9] data [10]. Mathematically, this is expressed as follows: If a function $f: R^{2} \rightarrow R$ with compact support has its Radon transform $\mathfrak{R}_{\theta} f(s)$ defined on the whole space $S^{1} \times[-1,1]$, say that the data is complete, otherwise we say that the data are incomplete. Recall that that is the unit circle.

\subsection{The Region of Interest}

An example of a problem of incomplete data is the interior problem, called in medical contexts the Region of Interest (ROI). This can be stated as follows: How to calculate the values of $f(x, y)$ for all $(x, y)$ satisfying $x^{2}+y^{2}=a^{2}$
, with, $0<a<1$ from the knowledge of projections of $f(x, y)$ on the lines passing through the neighborhood radius of the origin? Instead of know the Radon transform $\mathfrak{R}_{\theta} f(s)$ of $f(x, y)$ for all s in $S^{1} \times[-1,1]$, is known only in the restriction $S^{1} \times[-a, a]$ [9], [10]. The region of interest is not always in the center of the object; you can be anywhere in the object support. Mathematical analysis is performed assuming that usually it is in the center of the object [15], [16]. Figure 7 depicts sinograms two regions, one located on the source of the object, and the other outside the source.

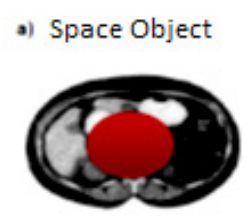

Radon Space
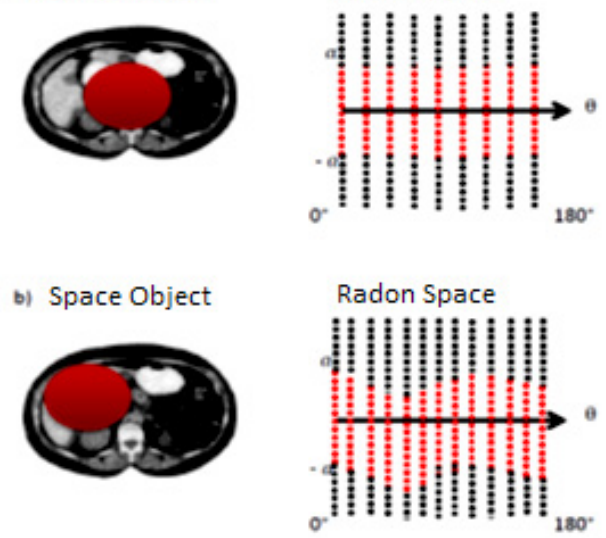

Figure 7. Sinograms a) ROI on the source of the object b) ROI outside source. Slice of abdomen. Source: own

Having little data is analogous to not having scanned the selected section of the patient completely. This may be perhaps because probably no one is interested in the full court all but a small portion (region of interest).

Benefits

- In medicine, in certain situations, the study is performed on a region of interest (ROI) so no need to expose the patient to radiation avoiding large amounts of long-term collateral damage to organs traversed by X-rays, such as heart, lungs and spinal injuries. 
VISION

ELECTRONICA
- In other sciences, images can be reconstructed with few samples obtained from non-destructive testing of the object as for example, in anthropological studies in forensic science, archeology and paleontology, among others.

Figure 8 a) illustrates local no problems tomographic data are obtained where the line integrals along all lines passing through the object; In practice this is done along a large number of lines with many angles. In problems of local data $b)$ the reductions appreciate the explanations offered by the scan [17], [18], [19].

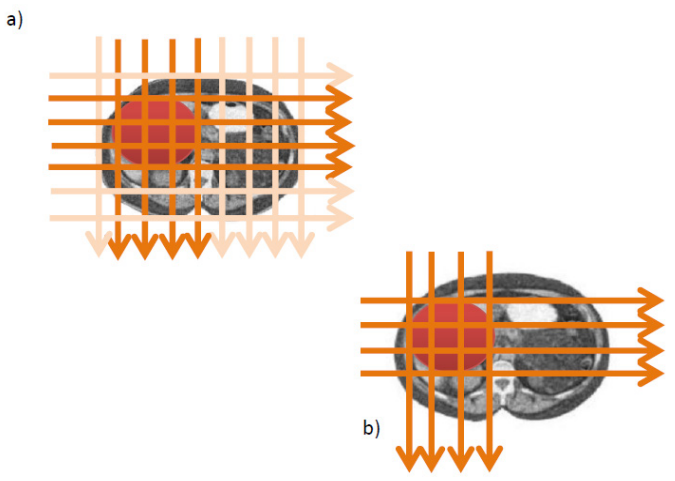

Figure 8. Data problems. a) Full, global or local. b) Incomplete or local. Slice of abdomen. Source: own

\subsection{The problem with local tomography}

Is it possible to reconstruct a tomographic image using only integrals that pass through the region of interest? : No, when the filtered back-projection algorithm is implemented with bases lacking now fading. Yes, when the filtered back-projection algorithm uses wavelet basis [18], [19], [20], [21], [22], [23].

\section{Wavelets Bases}

Recall that wavelets are localized smooth functions with compact support and a number of vanishing moments [24], [25], [26], [27],
[28]. Figure 9 shows the Haar wavelet, which has a compact support of one width, a time of fading and is discontinuous.

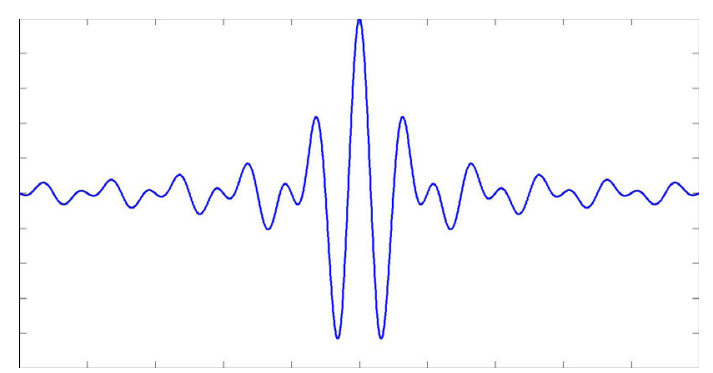

Figure 9. Haar Wavelet. Source: own

Figure 10 illustrates the Shannon wavelet, which is very soft but extends through the whole real line and decays slowly when time is infinite.

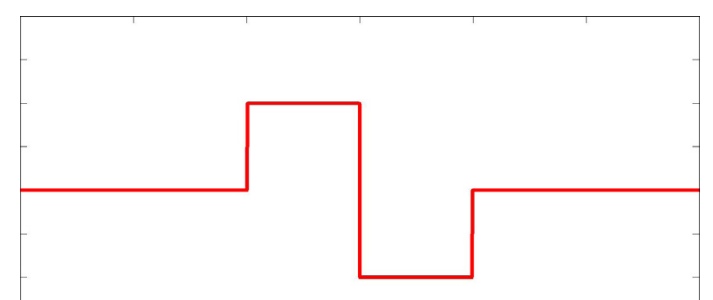

Figure 10. Shannon Wavelet. Source: own

The wavelets are generated from dilations and translations of a prototype function, called the mother wavelet by the equation:

$\psi^{a, b}(t)=\frac{1}{\sqrt{|a|}} \psi\left(\frac{t-b}{a}\right)$

Where $b \in R, a \in R_{+} \quad$ con $a \neq 0$ and $\psi$ is admissible [5]. With orthogonal wavelet bases, Riesz bases and frames for spaces of finite energy signals (like projections) are constructed. A result proved by David Walnut relates us smoothness of wavelets with vanishing moments. If the family $\left\{\psi_{j, k}\right\}$ is an orthonormal basis, and the wavelet is smooth, then it should have no or fade in time [29]. 


\subsection{Daubechies Bases}

In 1988, Ingrid Daubechies constructed a family of orthogonal wavelet basis, smooth and compactly supported. Daubechies wavelets, as they are called, are those with the highest number of vanishing moments for support. The width of the support is $2 N-1$ where $N$ the number of vanishing moments. Daubechies wavelets are classified according to the number of times of blackouts $N$ that they have [5], [30]. The Hilbert transform function works well with soft, especially with oscillatory wavelets. This can be checked with studies on the behavior of the Hilbert transform of wavelets [12], [31], [32], [33].

Some important conclusions for our purposes are:

- The Hilbert Transform $H \psi(x)$ of a wavelet $\psi$ is still a wavelet.

- In the presence of some minimal smoothness and decay, the Hilbert transform of a wavelet is smooth and oscillating as the original wavelet.

- The location can be controlled by the number of times of fading from the original wavelet.

- Decay of the Hilbert transform increases with increasing fading moments; many moments of lightheadedness may contribute to enhancing the Hilbert transform to $\frac{1}{\pi x^{n+1}}$ in the presence of $N$ fading moments [4].

- The Hilbert transform of a wavelet basis generated from the mother wavelet $\psi$ is the wavelet basis generated from the wavelet $H \psi(x)$.

\subsection{Concrete examples}

The figures 11 to 19 are concrete examples of the results discussed in section 5.1. The behavior of the location, the compact support, the smoothness and the moments of fadings Hilbert transform as it increases the degree of the Daubechies observed therein.

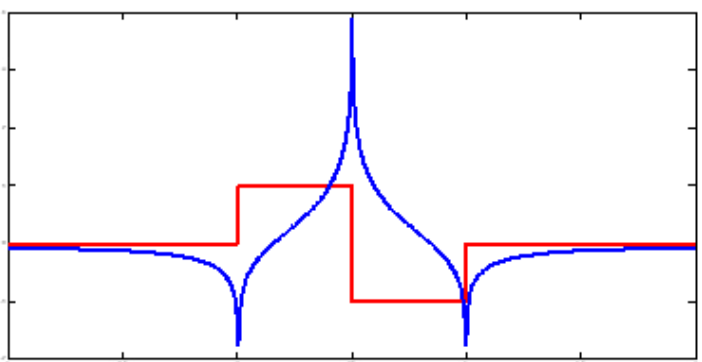

Figure 11 Daubechies Wavelet 1 (red) Hilbert Transform (blue). Source own

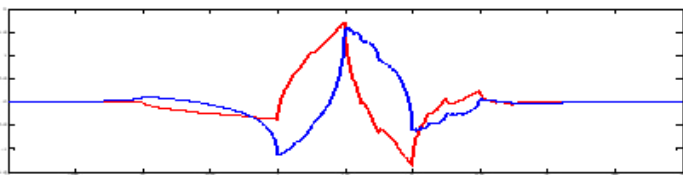

Figure 12 Daubechies Wavelet 2 (red) Hilbert Transform (blue). Source: own

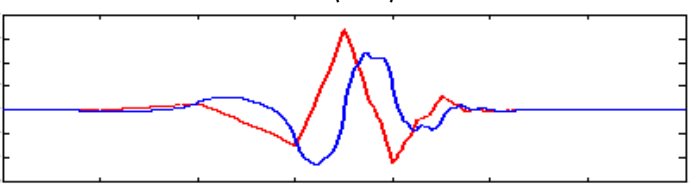

Figure 13 Daubechies Wavelet 3 (red) Hilbert Transform (blue). Source: own

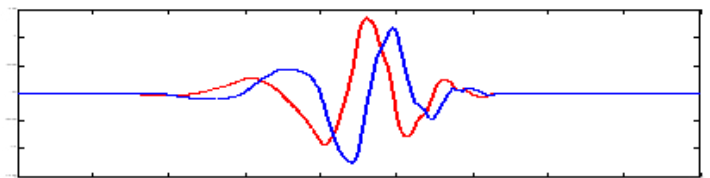

Figure 14 Daubechies Wavelet 4 (red) Hilbert Transform (blue). Source: own

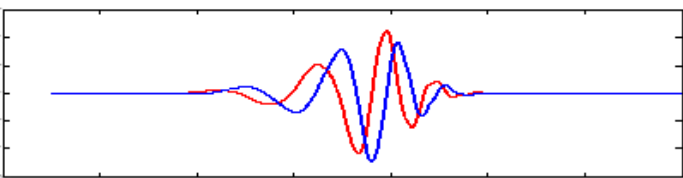

Figure 15 Daubechies Wavelet 6 (red) Hilbert Transform (blue). Source: own 


\section{VISION}

ELECTRONICA

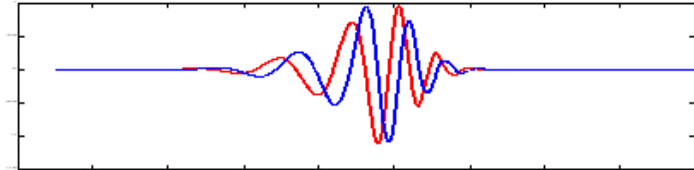

Figure 16 Daubechies Wavelet 8 (red) Hilbert Transform (blue). Source: own

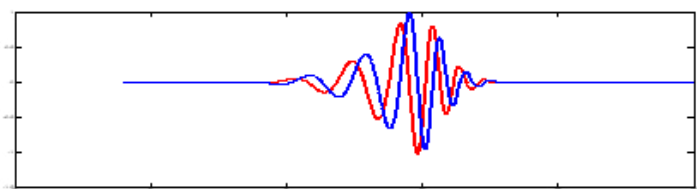

Figure 17 Daubechies Wavelet 10 (red) Hilbert Transform (blue). Source: own

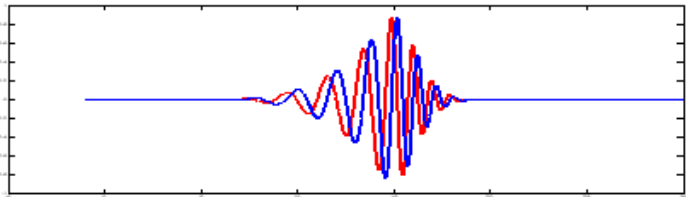

Figure 18 Daubechies Wavelet 15 (red) Hilbert Transform (blue). Source: own

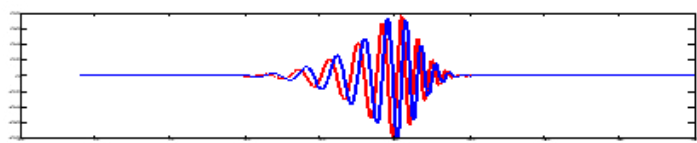

Figure 19 Daubechies Wavelet 20 (red) Hilbert Transform (blue). Source: own

Figures from 20 to 28 correspond to specific examples of Calderon-Zygmund operator and some Daubechies. The behavior of the spot, the compact support, smoothness and moments of fading as the operator increases the degree of observed Daubechies.

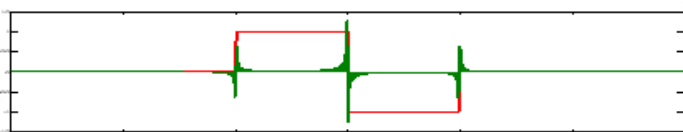

Figure 20 Daubechies Wavelet 1 (red) Calderón-Zygmund Operator (green). Source: own

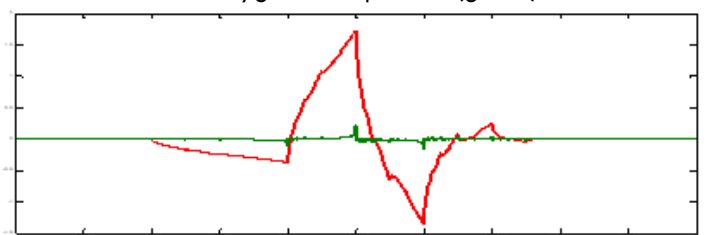

Figure 21 Daubechies Wavelet 2 (red) Calderón-Zygmund Operator (green). Source: own

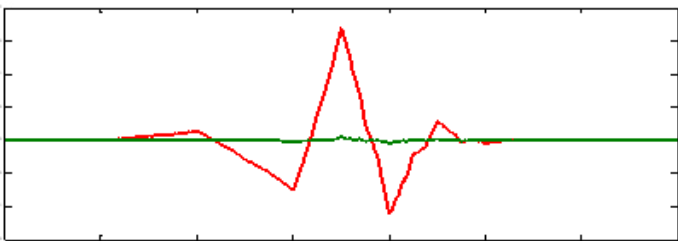

Figure 22 Daubechies Wavelet 3 (red) Calderón-Zygmund Operator (green). Source: own

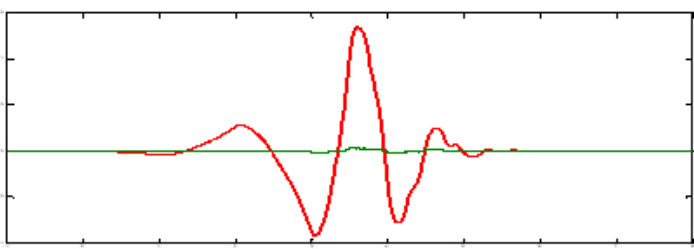

Figure 23 Daubechies Wavelet 4 (red) Calderón-Zygmund Operator (green). Source: own

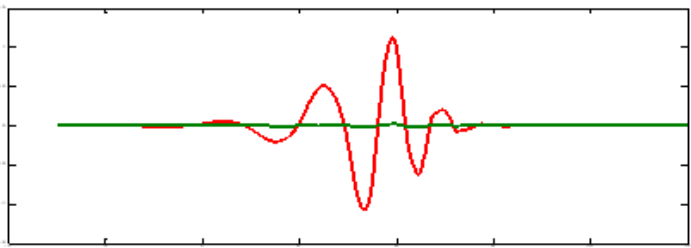

Figure 24 Daubechies Wavelet 6 (red) Calderón-Zygmund Operator (green). Source: own

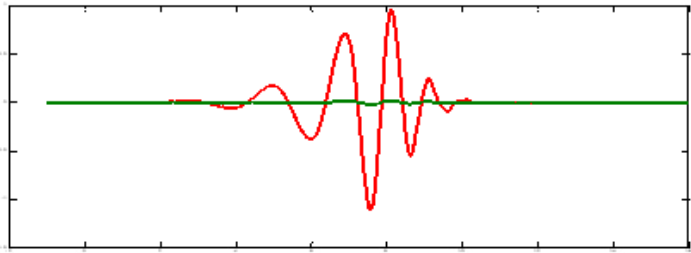

Figure 25 Daubechies Wavelet 8 (red) Calderón-Zygmund Operator (areen). Source: own

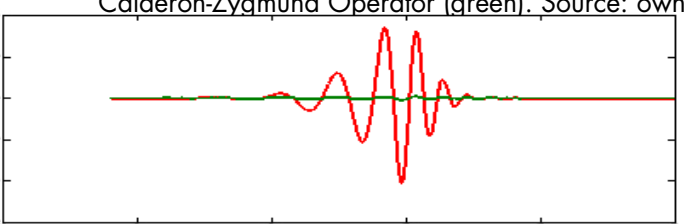

Figure 26 Daubechies Wavelet 10 (red) Calderón-Zygmund Operator (green). Source: own

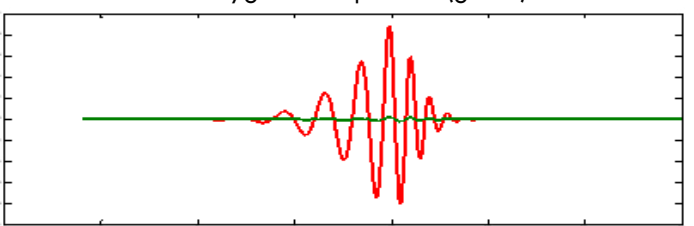

Figure 27 Daubechies Wavelet 15 (red) Calderón-Zygmund Operator (green). Source: own 


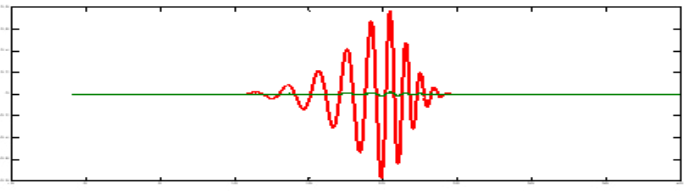

Figure 28 Daubechies Wavelet 20 (red) Calderón-Zygmund Operator (green). Source: own

\section{Conclusions}

The filtered back-projection algorithm used to invert the Radon transform presents problems if the bases that are deployed do not have sufficient time to blackouts. Studies like the one presented in this paper show that it is possible to obtain images using localized wavelet basis; and more specifically, Daubechies basis. These have a large number of times of fading for support, which causes the problem caused by the operator exceeds Calderon - Zygmund when convolved with the partial derivatives of the projections aren't necessary to use more of the data present. These bases have allowed the development of many algorithms for localized reconstructing images as can be seen in a more detailed study [34] or [37].

\section{Acknowledgements}

This work was funded by the Research Center for Innovation ICTP. Faculty of Engineering. Central University - Colombia.

\section{References}

[1] G.T. Herman, "Fundamentals of Computarized Tomography: Image Reconstruction from Projections (Advances in Pattern Recognition)", 2nd Ed, Springer. 2009.

[2] Ge. Wang, and M. W. Vannier, "Computarized Tomography”, Departament of Ra- diology, University of Iowa, Iowa 52242, Iowa City (USA). 1998.

[3] M. Defrise, and G. T. Gullberg, "Image Reconstruction”, Phys. Med. Biol. 51. 2006.

[4] K. Narayan, and M. Unser, "On the Hilbert Transform of Wavelets”, IEEE Transactions on signal processing, Vol. 59. no. 4. 2010.

[5] I. Daubechies, "Ten Lectures on Wavelets”, SIAM. 1992.

[6] W. A. Kalender, "Computed Tomography: Fundamentals, System Technology, Image Quality, Apllications”, Wiley. 2006.

[7] C. Kak, and M. Slaney, "Principles of Computarized Tomography”, New -york: IEEE press. 1988.

[8] S. R. Deans, “The Radon Transform and some of its aplications", Ed: John Wiley and Sons Inc., New York. 1983.

[9] F. Natterer, "The Mathematics of Computarized Tomography”, John Wiley\&Sons, New York, 1986.

[10] F. Natterer, "Fourier reconstruction in tomography”, Numer. Math. pp. 343-353. 1985.

[11] A. Markoe, "Fourier inversion of the attenuated Radon transform", SIAM J. Math. Anal. To appear. 1986.

[12] E. Bedrosian, "A product theorem for Hilbert transform" Proc. IEEE. Vol. 51. no. 5. 1963.

[13] M. Bottema, B. Moran, and S. Suvorova "An Apllication of Wavelets in To- 
R. De Armas, A. Alfonso y L. Rojas,

VISION

ELECTRONICA mography”, Digital Signal Processing 8. SP980326. 1998.

[14] G. B. Folland, "Fourier Analysis and Its Applications", Wadsworth\&Brooks/ Cole, Pacific Grove, CA, 1992.

[15] P. Kuchment, K. Lancaster and L. Magilezskaya, "On the structure of local tomography. Inverse Problems To appear”.

[16] P. Maass, "The interior Radon transform”, SIAM J. Appl. Math., Vol. 52, no. 3, pp. 70-724, 1992.

[17] K.T.Smith, "Inversion of the X-ray transform”,SIAM-AMS Proceedings,14, pp.41-52. 1984.

[18] C. Berenstein and D. Walnut, "Local inversion of the Radon transform in even dimensions using wavelets", In 75 Years of Radon Transform, S. Gindlikin and P. Michor, editors, 45-69. International Press, Cambridge, MA, 1994.

[19] A. Faridani, D. Finch, E. Ritman, and K. Smith, "Local tomography II", SIAM J. Appl. Math., vol. 57, n0.4, 2006, DOI: https://doi.org/10.1137/ S0036139995286357.

[20] A. Faridani, F. Keinert, F. Natterrer, E. Ritman, and K. Smith, "Local and tomography. In Signal Processing”, IMA Vol. Math. Apll., Vol. 23, Springer-Verlag, New York, pp. 241-255. 1990.

[21] M. Holschneider, "Inverse Radon transforms. Inverse problems”, pp. 853-861, 1999.

[22] A. Faridani, E. Ritman, and K. T. Smith, "Local tomography", SIAM J Appl. Math., Vol. 52, no. 2, pp. 459-484, April 1992.
[23] Louis, A. ,K., and A. Reider, "Incomplete data problems in X- Ray computerized tomography”, Numeriche Mathemetik, 56, pp. 371- 383.1989,

[24] Chui, C., "An Introduction to Wavelets", Vol. 1-2, Academic Press, San Diego, 1992.

[25] Mallat, S., "A Wavelet Tour of Signal Processing", Academic Press, San Diego, 1998.

[26] Meyer, Y., "Wavelets \& Applications, Society for Industrial and Applied Mathematics”, Philadelphia, PA, 1993.

[27] R. S. Strichartz, "How To Make Wavelets," Amer. Math. Monthly, 100 pp. 539556. 1993.

[28] Albert Boggess., and Francis J. Narcowitch, "A First Course in Wavelets with Fourier Analysis". Prentice-Hall, Inc. 2001.

[29] D. Walnut, "An Introduction to Wavelet Analysis”, Birkhauser. 2001.

[30] I. Daubechies, "Orthonormal bases of compactly supported wavelets II: Variations on a theme", SIAM J. Math. Anal 24. 1993.

[31] C. Chaux, L. Duval, J.C. Pesquet, "Hilbert pairs of $\mathrm{m}$-band orthonormal wavelet bases", in Proc. Eur. Signal Image Process. Conf. 2004.

[32] K. N. Chaudhury, and M. Unser "Construction of Hilbert transform pairs of wavelet bases and Gabor-like transforms," IEEE Trans. Signal Process., Vol 57. no. 9. 2009. 
[33] T. Olson, and J. DeStefano, "Wavelet localization of the Radon transform" IEEE Trans. Signal Process. Vol 42. no. 8. 1994.

[34] J. Villegas, A. Alfonso, R. De Armas, "El problema de la tomografía local. Enfoque wavelets”, Universidad Central. Bogotá. 2012 .

[35] F. R. Kschischang, "The Hilbert Transform", The Edward S. Rogers Sr. Departament of Electrical and Computer
Engineering University of Toronto. October 22 .

[36] S. L. Halnn, "Hilbert Transforms", in The Transforms and Applications Handbook (A. Poularakis, Ed.) Boca Raton Fl: CRC Press, ch 7. 1996.

[37] H. Vacca, W. Díaz. "Problema de tomografía local usando Wavelets B-Spline Cúbicos". Revista Tecnura. Edición especial, 13-29. 2014. 